Research Article

\title{
Correlation between Handgrip Strength and Rapid Shallow Breathing Index for Assessment of Weaning from Mechanical Ventilation
}

\author{
Narongkorn Saiphoklang (iD) and Thanapon Keawon \\ Division of Pulmonary and Critical Care Medicine, Department of Internal Medicine, Faculty of Medicine, \\ Thammasat University, Bangkok, Thailand
}

Correspondence should be addressed to Narongkorn Saiphoklang; m_narongkorn@hotmail.com

Received 29 August 2021; Accepted 20 November 2021; Published 30 November 2021

Academic Editor: Quincy K Tran

Copyright (c) 2021 Narongkorn Saiphoklang and Thanapon Keawon. This is an open access article distributed under the Creative Commons Attribution License, which permits unrestricted use, distribution, and reproduction in any medium, provided the original work is properly cited.

\begin{abstract}
Background. Assessment of weaning from mechanical ventilation (MV) is an important process. Rapid shallow breathing index (RSBI) is a standard tool to evaluate a patient's readiness before the spontaneous breathing trial (SBT). Handgrip strength (HGS) is an alternative method for assessment of respiratory muscle strength. Relationship between HGS and RSBI has not been explored. This study aimed to determine the correlation between HGS and RSBI to predict successful extubation in mechanically ventilated patients. Methods. A prospective study was conducted in screened 120 patients requiring MV with tracheal intubation $>48 \mathrm{~h}$. HGS was performed at $48 \mathrm{~h}$ after intubation, $10 \mathrm{~min}$ before and $30 \mathrm{~min}$ after SBT, and $1 \mathrm{~h}$ after extubation. RSBI was performed at 10 min before SBT. Results. A total of 93 patients $(58 \%$ men) were included in the final analysis. Mean age was $71.6 \pm 15.2$ years. Patients admitted in general medical wards were $84.9 \%$. APACHE II score was $13.5 \pm 4.7$. Most patients were intubated from pneumonia (39.8\%). Weaning failure was 6.5\%. The main result shows that HGS was negatively correlated with RSBI (regression coefficient $-0.571, P<0.001)$. The equation for predicting RSBI, derived from the linear regression model, was predicted RSBI (breaths $/ \mathrm{min} / \mathrm{L})=39.285+($ age $* 0.138)-($ HGS $* 0.571)$. Conclusions. HGS had significantly negative correlation with RSBI for assessment of weaning from MV. A prospective study of the HGS cutoff value is needed to investigate the difference between patients who succeeded and those who failed extubation. This trial is registered with TCTR20180323004.
\end{abstract}

\section{Introduction}

Most patients require mechanical ventilation (MV) with intubation due to respiratory failure. Assessment of their readiness for ventilator weaning is a crucial approach to reduce complications and mortality [1].

The use of rapid shallow breathing index (RSBI) has increased widely due to its simple method and avoidance of calculation of complex pulmonary mechanics [2]. It is the most accurate predictor of failure in weaning patients from MV [3] and is the most frequently studied and seems to be an important measurement tool in deciding whether to wean or extubate a patient [4]. However, the results of several studies revealed that weaning and extubation should be guided by several parameters and not only by respiratory ones [4]. Besides RSBI, several parameters can predict weaning success, e.g., handgrip strength (HGS), heart rate variability, sleep quality, diaphragmatic dysfunction, and biomarkers [5]. HGS may be a useful tool for the weaning assessment from prolonged MV at a long-term acute-care hospital, in addition to the maximum inspiratory pressure (PImax) [6]. During 6-month follow-up, HGS increased 6.2 times compared to HGS at recruitment. During the same period, PImax also increased but to a lesser degree [6]. Moreover, hand grasping and tongue protrusion used as simple motor tasks were predictors of failed extubation in critically ill neurologic patients if they could not follow simple motor commands [7]. HGS can predict difficult or prolonged weaning in mechanically ventilated patients, supported by a previous study that showed different HGS 
between weaning groups: $20 \mathrm{~kg}, 12 \mathrm{~kg}$, and $6 \mathrm{~kg}$ for simple, difficult, and prolonged weaning, respectively [8]. Low strength was correlated with significantly increased reintubation rate [9]. In the previous study, patients in the reintubation group had significantly lower HGS at $10 \mathrm{~min}$ and $30 \mathrm{~min}$ after starting spontaneous breathing trial (SBT) $(7.6 \pm 4.8 \mathrm{~kg}$ vs. $13.4 \pm 6.5 \mathrm{~kg}$ and $8 \pm 5.1 \mathrm{~kg}$ vs. $13.2 \pm 5.7 \mathrm{~kg})$ compared to the non-reintubation group [9]. Furthermore, intensive care unit-acquired paresis was associated with lower HGS, poor hospital outcomes, and in-hospital mortality [10]. HGS was also correlated with respiratory muscle strength in the healthy elderly, as well as the maximal inspiratory pressure and maximal expiratory pressure [11].

We hypothesized that HGS indicates respiratory muscle strength and may be correlated with RSBI, an established predictor of successful extubation. This study aimed to evaluate the correlation between HGS and RSBI for weaning from MV.

\section{Materials and Methods}

2.1. Study Design and Subjects. A prospective study was conducted between January 2018 and January 2020 in general medical wards and a medical intensive care unit (ICU) at Thammasat University Hospital, Thailand. Patients requiring $\mathrm{MV}$ with tracheal tube intubation for at least $48 \mathrm{~h}$ and aged 18 years or older were recruited. All recruits were able to cooperate fully, able to do the HGS test, and were expected to pass the SBT upon extubation from MV. Exclusion criteria were death before weaning from MV, less than $48 \mathrm{~h}$ of MV, transfer to other hospitals, self-extubation, accidental extubation, reintubation before enrollment, undergoing tracheostomy, inability to perform the HGS test, and treatment with vasopressor/inotropic drugs. The 34 patients successfully recruited for this study were also among the patients who participated at the same time in our previously published study [9].

The weaning process was under the supervision of the attending physicians. However, all members of the team who participated in the weaning process underwent prestudy training on weaning according to the standards of the European and American Respiratory/Intensive Care Societies [1]. Weaning was attempted as early as possible during the patients' illnesses with a two-step approach in which readiness for weaning was assessed daily according to the standard criteria of the European and American Respiratory/Intensive Care Societies [1]. Patients who fulfilled these criteria underwent SBT. The duration of SBT was 30-120 min and consisted of either breathing with a T-piece or a weaning trial undergoing $5-8 \mathrm{~cm} \mathrm{H}_{2} \mathrm{O}$ pressure support with $5 \mathrm{~cm} \mathrm{H}_{2} \mathrm{O}$-positive end-expiratory pressure. When patients successfully passed the SBT, the physician in charge, in collaboration with the attending medical staff initiated the weaning process. If a patient failed the initial SBT, MV was reinstituted, and the physician reviewed the possible reversible causes of the weaning failure, e.g., bronchospasm, secretion obstruction, pulmonary edema, congestive heart failure, psychoneurological factors, electrolyte imbalances, malnutrition, and anemia. SBT was repeated the following day if the patient then appeared ready for weaning. A patient was rated as successfully weaned when he or she was extubated and breathing spontaneously without any invasive or noninvasive ventilatory support for $\geq 48 \mathrm{~h}$. Concordantly, weaning failure was defined as either the failure of SBT or the need for reintubation within $48 \mathrm{~h}$ following extubation [1].

Participants were classified into 3 weaning groups: (1) simple weaning: successful weaning and extubation on the first attempt without difficulty, (2) difficult weaning: failure of initial weaning and the need for up to three SBTs for as many as 7 days from the first SBT to achieve successful weaning, and (3) prolonged weaning: failure of 3 or more weaning attempts or the need for longer than 7 days of weaning after the first SBT. [1].

Demographics and baseline characteristics including acute physiology and chronic health evaluation (APACHE) II scores were collected for all participants. During SBT, data on MV weaning were collected including vital signs, RSBI, type of SBT, time of SBT success, and time of extubation. HGS was tested at $48 \mathrm{~h}$ after intubation and $10 \mathrm{~min}$ and $30 \mathrm{~min}$ after SBT. In addition, this measurement was performed at $1 \mathrm{~h}$ after extubation. RSBI and HGS test were both performed at $10 \mathrm{~min}$ before SBT. The physician in charge did not know HGS results.

Ethic approval was obtained from the Human Ethics Committee of Thammasat University (IRB No. MTU-ECIM-0-198/60), in compliance with Declaration of Helsinki, The Belmont Report, CIOMS Guidelines, and The International Practice (ICH-GCP). All methods were performed in accordance with these guidelines and regulations. All participants provided written informed consent.

2.2. Outcomes and Measurements. Primary outcome was the association between HGS and RSBI, adjusted for age, sex, and APACHE II score. Secondary outcomes were the difference in HGS between weaning success and weaning failure groups and the differences in HGS between simple, difficult, or prolonged weaning groups. Maximal grip strength from 3 efforts for each hand was recorded using a specialized dynamometer (Jamar, Asimow Engineering Co., Santa Monica, CA, USA), The measurements were made at rest with the hand unsupported, with the elbow at $90^{\circ}$ flexion, and with the wrist in neutral position. RSBI was calculated by patient's respiratory rate divided by tidal volume (breaths/min/L), which were obtained while the patient was undergoing $\mathrm{MV}$ with $5 \mathrm{~cm} \mathrm{H}_{2} \mathrm{O}$ positive endexpiratory pressure and no pressure support.

2.3. Statistical Analysis. The sample size was calculated on the basis of our hypothesis expecting the correlation coefficient between HGS and RSBI of 0.5 . Thus, 38 patients would provide a power of $90 \%$ and a two-sided alpha level of $5 \%$. Categorical data were shown as number (\%). Continuous data were shown as mean \pm standard deviation. Student' $t$-test was used to compare continuous variables between two groups. ANOVA was used for comparison of 3 weaning groups. To determine the set of variables associated with RSBI, we used the linear regression model with the RSBI set as dependent variable. All independent variables (age, sex, HGS, and APACHE II score) 
were entered into the regression model simultaneously. We report the regression coefficients, their 95\% confidence interval, and corresponding $p$ values. Variables with $p$ value $<0.05$ were considered statistically associated with RSBI. Using the regression coefficients and the intercept, predicted RSBI for a patient could be calculated from the following equation, where $V$ represents the covariate, $\beta$ represents the regression coefficient, and $i$ represents the number of variables:

$$
\text { Predicted RSBI }=\text { intercept }+V 1 \beta 1+V 2 \beta 2+V i \beta i .
$$

Statistical analyses were performed using SPSS version 20.0 software (IBM Corp., Armonk, NY, USA).

\section{Results}

3.1. Patients. One hundred twenty mechanically ventilated patients were recruited, and 93 of these were included in the final analysis (Figure 1). There were 54 (58.1\%) males. Mean age was $71.6 \pm 15.2$ years. Most patients were admitted in general medical wards (84.9\%, 79 of 93 patients). APACHE II score was $13.5 \pm 4.7$. Most patients were intubated from pneumonia (39.8\%, 37 of 93 patients). The most common type of SBT was pressure support ventilation $(74.2 \%, 69$ of 93 patients) (Table 1). The highest HGS was at $1 \mathrm{~h}$ after extubation $(16.3 \pm 6.5 \mathrm{~kg})$. RSBI at $10 \mathrm{~min}$ before SBT was $40.2 \pm 9.2$ breaths $/ \mathrm{min} / \mathrm{L}$. Weaning failure was $6.5 \%$ (6 of 93 patients) (Table 2). The causes for extubation failure were pneumonia $(33.3 \%, 2$ of 6 patients), pulmonary edema (16.7\%, 1 of 6 patients), marked airway secretions (16.7\%, 1 of 6 patients), bronchospasm (16.7\%, 1 of 6 patients), and hypoalbuminemia (16.7\%, 1 of 6 patients). Incidence of simple, difficult, and prolonged weaning was $77.4 \%, 20.4 \%$, and $2.2 \%(72,19$, and 2 of 93 patients), respectively.

3.2. Primary Outcome. The main result shows that HGS was negatively correlated with RSBI. The equation for predicting RSBI, derived from the linear regression model, was predicted RSBI (breaths $/ \mathrm{min} / \mathrm{L})=39.285+($ age $* 0.138)-(\mathrm{HGS} *$ 0.571 ), with regression coefficient $-0.571, P<0.001$ (Figure 2 and Table 3).

3.3. Secondary Outcome. HGS was significantly higher in patients in the weaning success group than in the weaning failure group over time. However, RSBI did not significantly differ between the two groups (Table 2).

At $10 \mathrm{~min}$ before SBT in simple, difficult, and prolonged weaning groups, maximum HGS was $16.3 \pm 7.2,13.7 \pm 4.7$, and $15.1 \pm 0.4 \mathrm{~kg}$, respectively $(P=0.202)$, and RSBI was $39.3 \pm 9.7,43.6 \pm 6.7$, and $39.0 \pm 9.2$ breaths $/ \mathrm{min} / \mathrm{L}$, respectively $(P=0.318)$.

For predicting successful extubation, the best HGS cutoff value at $10 \mathrm{~min}$ before SBT was $12.7 \mathrm{~kg}$ with area under the receiver operating characteristic (ROC) curve of 0.842 (95\% CI: $0.67-1.00, P=0.005)$. We observed a $75.9 \%$ sensitivity and $83.3 \%$ specificity for the $12.7 \mathrm{~kg}$ HGS cutoff. The best RSBI cutoff value at 10 min before SBT was 43.5 breath$\mathrm{s} / \mathrm{min} / \mathrm{L}$, with area under the ROC curve of 0.459 (95\% CI: $0.16-0.75, P=0.737), 33.3 \%$ sensitivity, and $66.6 \%$ specificity.

\section{Discussion}

This study is the first prospective study that revealed the correlation between HGS and RSBI to predict successful extubation in mechanically ventilated patients. It differs from our previously published study [9] which showed that low HGS was correlated with the significantly increased reintubation rate in mechanically ventilated patients. The 34 patients recruited for this current study also participated in our previously published study at the same time. Majority of our patients (85\%) were admitted in general medical wards due to the limitation of ICU beds in our hospital. However, there were sufficient devices for respiratory and noninvasive hemodynamic monitoring in general medical wards. Our research with patients in a mixed population from general medical wards and a medical ICU revealed that HGS had a significantly negative correlation with RSBI for assessment of the weaning process. Furthermore, this study derived the equation predicting RSBI by using the HGS factor to determine the weaning outcome. Several studies had explored HGS in critically ill patients receiving MV, but these studies did not investigate the relationship between RSBI and HGS $[7,10,12]$.

A study by Yang and Tobin determined that RSBI is an accurate index to predict weaning failure (a threshold of 105 breaths/min/L with $78 \%$ positive predictive value and $95 \%$ negative predictive value) which is more accurate than CROP index (dynamic compliance, respiratory rate, oxygenation, and maximum inspiratory pressure index), minute ventilation, or maximal inspiratory pressure [3]. However, some patients will require reintubation and institution of MV despite meeting established weaning criteria, while some patients not meeting criteria can be successfully liberated from the ventilator. Our RSBI was smaller, 70 breaths/ min/L, than in Yang and Tobin' study [3], which may have resulted from the fact that most of our patients were elderly (mean aged 71 years old) and some patients had COPD; thus, these patients might have had air trapping and high lung volume leading to lower RSBI. Moreover, RSBI did not differ between those who were successful in extubation and those that failed in our study. We speculated that the RSBI values could have been confounded by factors other than respiratory muscle strength (e.g., increased respiratory rates from pneumonia and low tidal volume in pulmonary edema), leading to misleading results. Meanwhile, HGS is directly correlated with respiratory muscle strength with less interference from other factors.

Mohamed-Hussein AAR et al., in a study comparing HGS at weaning to intensive care outcomes in 34 patients with chronic obstructive pulmonary disease (COPD), found that HGS may be a good predictor for extubation outcomes as well as MV duration and ICU mortality [12].

A previous study of Cottereau $G$ et al. demonstrated that there were statistically significant differences in HGS between simple, difficult, and prolonged weaning groups $(20 \mathrm{~kg}, 12 \mathrm{~kg}$, and $6 \mathrm{~kg}$, respectively, $P=0.008$ ), but no association was found between HGS and extubation failure [8]. A previous study by Saiphoklang and Auttajaroon showed that there were 


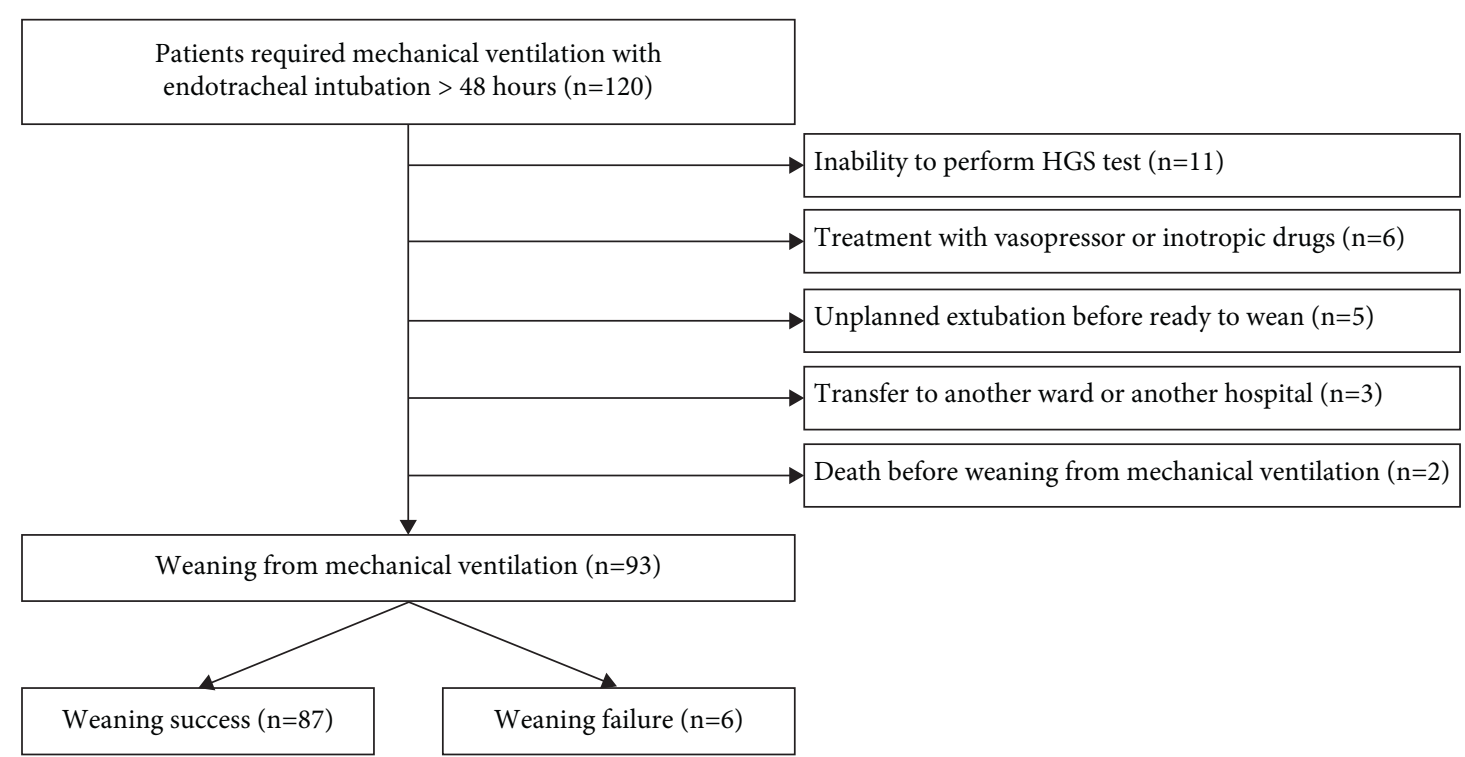

Figure 1: Study flowchart indicating inclusion and exclusion population.

TABLE 1: Baseline characteristics.

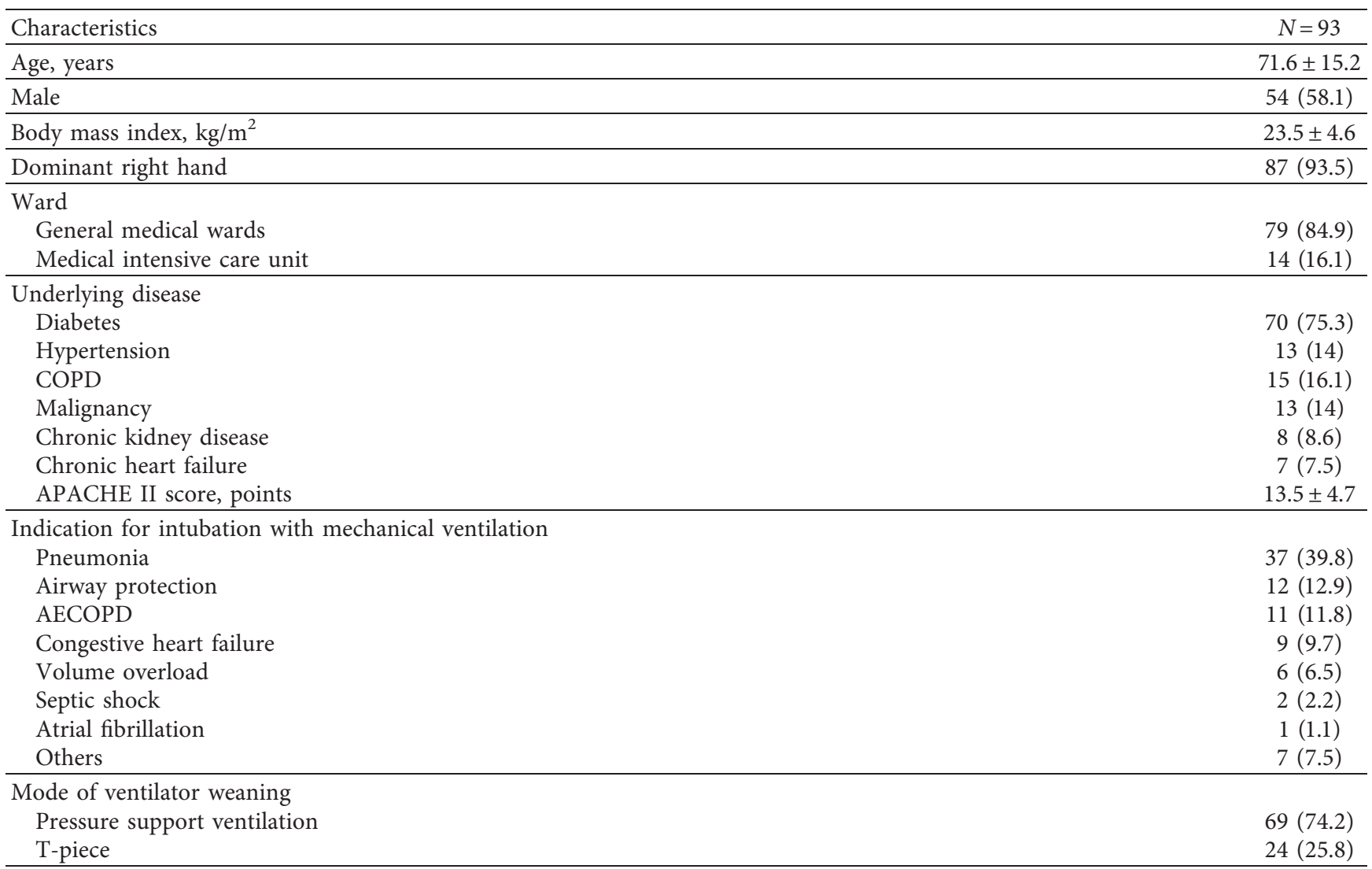

Data are presented as $n(\%)$ or mean \pm SD. AECOPD, acute exacerbation of COPD; APACHE II, acute physiology and chronic health evaluation II; COPD, chronic obstructive pulmonary disease.

significant differences in mortality rates between 3 weaning groups, but HGS was not investigated [13]. In contrast, our study shows there was no difference in HGS between the 3 weaning groups, but there was a significant difference in HGS between weaning success and weaning failure groups. The first reason for the findings may be because the previous study [8] was conducted only in ICU which needed more intensive and close monitoring after weaning or extubation such as airway care and pulmonary rehabilitation, unlike this study which included over $50 \%$ of patients from general medical wards. Another reason is that our patients in this study were recruited from a more aging population (mean age of 71 years), and the 
TABLE 2: Comparison of handgrip strength and rapid shallow breathing index between weaning success and weaning failure.

\begin{tabular}{|c|c|c|c|c|}
\hline Variable & Total, $n=93$ & Weaning success, $n=87$ & Weaning failure, $n=6$ & $P$ value \\
\hline HGS at $48 \mathrm{~h}$ after intubation, $\mathrm{kg}$ & $15.6 \pm 7.0$ & $16.2 \pm 7.3$ & $6.8 \pm 4.7$ & 0.002 \\
\hline HGS at $10 \mathrm{~min}$ before SBT, $\mathrm{kg}$ & $15.8 \pm 6.7$ & $16.3 \pm 6.5$ & $8.2 \pm 5.3$ & 0.004 \\
\hline HGS at $30 \mathrm{~min}$ before SBT, $\mathrm{kg}$ & $15.9 \pm 6.6$ & $16.4 \pm 6.3$ & $8.6 \pm 5.6$ & 0.004 \\
\hline HGS $1 \mathrm{~h}$ after extubation, $\mathrm{kg}$ & $16.3 \pm 6.5$ & $16.8 \pm 6.3$ & $8.6 \pm 5.5$ & 0.002 \\
\hline RSBI at $10 \mathrm{~min}$ before SBT, breaths/min/L & $40.2 \pm 9.2$ & $40.0 \pm 8.9$ & $42.2 \pm 14.4$ & 0.590 \\
\hline
\end{tabular}

Data are presented as mean \pm SD. HGS, handgrip strength; RSBI, rapid shallow breathing index; SBT, spontaneous breathing trial.

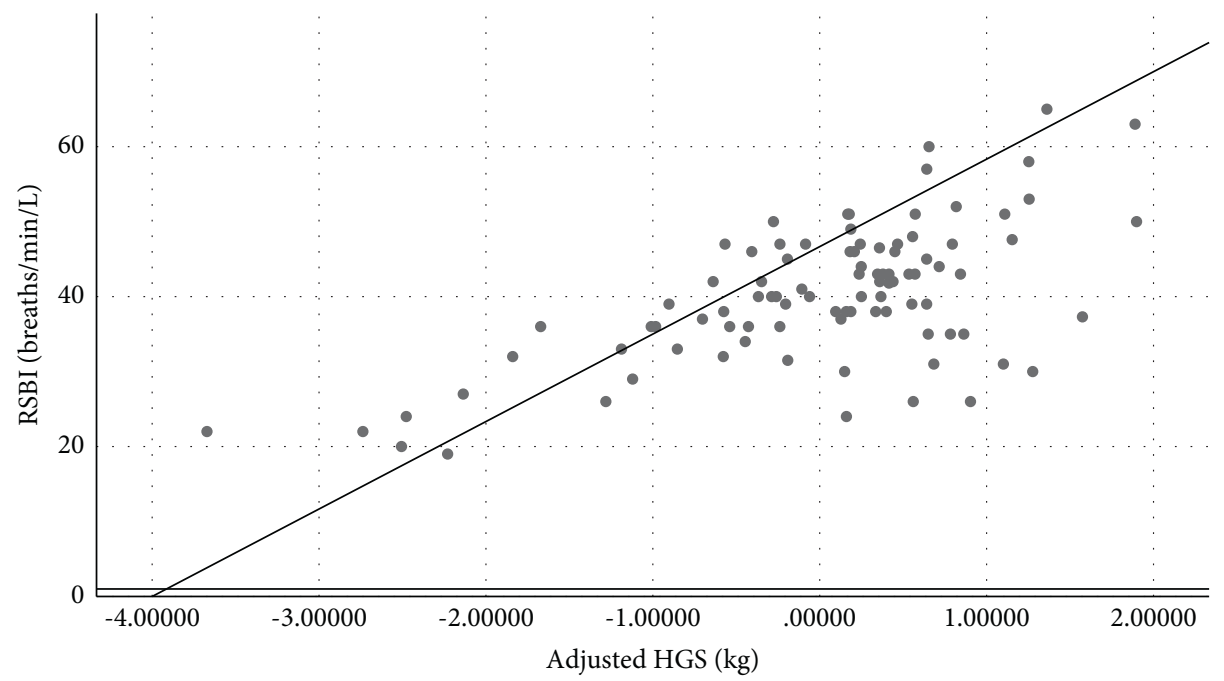

FIGURE 2: Linear regression analysis showing the correlation between rapid shallow breathing index (RSBI) and handgrip strength (HGS) at $10 \mathrm{~min}$ before spontaneous breathing trial (SBT) adjusted by age, gender, and acute physiology and chronic health evaluation II score. Predicted RSBI $=39.285+($ age $* 0.138)-($ HGS $* 0.571)$, regression coefficient: $-0.571, P<0.001$.

TABLE 3: Linear regression analysis for rapid shallow breathing index and handgrip strength, age, gender, and APACHE II score.

\begin{tabular}{lccc}
\hline Variables & Regression coefficients & $95 \%$ CI of coefficients & $P$ value \\
\hline Intercept & 39.285 & $27.491,51.079$ & $<0.001$ \\
HGS at 10 min before SBT & -0.571 & $-0.834,-0.309$ & $<0.001$ \\
Age & 0.138 & $0.033,0.242$ & 0.010 \\
Male & -3.119 & $-6.466,0.229$ & 0.067 \\
APACHE II score & 0.138 & $-0.215,0.491$ & 0.439 \\
\hline
\end{tabular}

HGS, handgrip strength; SBT, spontaneous breathing trial.

main indications for MV were respiratory conditions such as pneumonia, airway protection, and COPD exacerbation, which may have had direct effects on weaning processes.

This study has a few limitations. The first is that this study cannot demonstrate the cut point of HGS for extubation failure. The second limitation is limited postextubation monitoring in general wards as discussed above. The last is that this study included only patients from medical wards and thus may not apply to surgical patients. A future study may find the cut point of HGS of extubation success in order to implement it to a ventilator liberation protocol.

\section{Conclusions}

HGS had significantly negative correlation with RSBI for assessment of the weaning process in mechanically ventilated patients. A prospective study of the HGS cutoff value is needed to investigate the difference between patients who succeeded and those who failed extubation.

\section{Data Availability}

The data used to support the results of this study are included within the article.

\section{Conflicts of Interest}

The authors declare that they have no conflicts of interest.

\section{Authors' Contributions}

All authors participated in the design of this analysis, data collection and analysis, manuscript writing, and revision.

\section{Acknowledgments}

The authors would like to thank Michael Jan Everts who is a lecturer at Faculty of Medicine in Thammasat University for proofreading this manuscript. The financial support was provided by Faculty of Medicine, Thammasat University, Thailand. 


\section{References}

[1] J.-M. Boles, J. Bion, A. Connors et al., "Weaning from mechanical ventilation," European Respiratory Journal, vol. 29, no. 5, pp. 1033-1056, 2007.

[2] M. Karthika, F. A. Al Enezi, L. V. Pillai, and Y. M. Arabi, "Rapid shallow breathing index," Annals of Thoracic Medicine, vol. 11, no. 3, pp. 167-176, 2016.

[3] K. L. Yang and M. J. Tobin, "A prospective study of indexes predicting the outcome of trials of weaning from mechanical ventilation," New England Journal of Medicine, vol. 324, no. 21, pp. 1445-1450, 1991.

[4] A. R. Baptistella, F. J. Sarmento, K. R. da Silva et al., "Predictive factors of weaning from mechanical ventilation and extubation outcome: a systematic review," Journal of Critical Care, vol. 48, pp. 56-62, 2018.

[5] H. Zein, A. Baratloo, A. Negida, and S. Safari, "Ventilator weaning and spontaneous breathing trials; an educational review," Emergency, vol. 4, no. 2, pp. 65-71, 2016.

[6] A. Jubran, B. J. B. Grant, L. A. Duffner et al., "Long-term outcome after prolonged mechanical ventilation. A long-term acute-care hospital study," American Journal of Respiratory and Critical Care Medicine, vol. 199, no. 12, pp. 1508-1516, 2019.

[7] F. M. Kutchak, M. D. M. Rieder, J. A. Victorino et al., "Simple motor tasks independently predict extubation failure in critically ill neurological patients," Jornal Brasileiro de Pneumologia, vol. 43, no. 3, pp. 183-189, 2017.

[8] G. Cottereau, M. Dres, A. Avenel et al., "Handgrip strength predicts difficult weaning but not extubation failure in mechanically ventilated subjects," Respiratory Care, vol. 60, no. 8, pp. 1097-1104, 2015.

[9] N. Saiphoklang and C. Tepwimonpetkun, "Interest of hand grip strength to predict outcome in mechanically ventilated patients," Heart \& Lung, vol. 49, no. 5, pp. 637-640, 2020.

[10] N. A. Ali, J. M. O’Brien Jr., S. P. Hoffmann et al., “Acquired weakness, handgrip strength, and mortality in critically ill patients," American Journal of Respiratory and Critical Care Medicine, vol. 178, no. 3, pp. 261-268, 2008.

[11] H. I. Shin, D.-K. Kim, K. M. Seo, S. H. Kang, S. Y. Lee, and S. Son, "Relation between respiratory muscle strength and skeletal muscle mass and hand grip strength in the healthy elderly," Annals of Rehabilitation Medicine, vol. 41, no. 4, pp. 686-692, 2017.

[12] A. A. R. Mohamed-Hussein, H. A. Makhlouf, Z. I. Selim, and W. Gamaleldin Saleh, "Association between hand grip strength with weaning and intensive care outcomes in COPD patients: a pilot study," The Clinical Respiratory Journal, vol. 12, no. 10, pp. 2475-2479, 2018.

[13] N. Saiphoklang and J. Auttajaroon, "Incidence and outcome of weaning from mechanical ventilation in medical wards at Thammasat University Hospital," PLoS One, vol. 13, no. 10, Article ID e0205106, 2018. 